\title{
Vorwort
}

Nach der für die Neuzeit gültigen Definition von „Kolonie“, in der exklusive Ansprüche auf die politisch-wirtschaftliche Eroberung der Kolonie von einem „Mutterland“ erhoben oder/und realisiert wurden, war China keine Kolonie Europas. Die „halbkoloniale“ und „halbfeudale“ Gesellschaft gibt die vorherrschende Idee von der Darstellung der Gesellschaftsformation Chinas in der Zeit des Kolonialismus wieder. Aber „Kolonialismus“ bezieht sich nicht nur auf den Souveränitätsverlust der Kolonie, sondern er ist auch ein Aufbauprozess der kulturellen Hegemonie, der so verlief, dass westliche Mächte mit mehr oder weniger Zwang die Bevölkerungen in den eroberten Gebieten dazu nötigten, die westlichen kulturellen Ideen und Modelle zu akzeptieren. Aus dieser Perspektive betrachtet erfuhr China ab dem Opiumkrieg 1840-1842 etwas strukturell Ähnliches wie die sogenannten typischen Kolonien und kann in die Forschung zur Geschichte des Kolonialismus mit einbezogen werden. Deshalb werden in der vorliegenden Studie die Wörter „halbfeudal“ und „halbkolonial“ nur bei der Beschreibung der Gesellschaftsformation Chinas angewandt und in anderen Fällen werden die betreffenden Begriffe des „Kolonialismus“ benutzt.

In den über die ganze Welt verstreuten Kolonien wurde die Kolonialherrschaft häufig durch die Einrichtung des medizinisch-hygienischen Systems gefestigt und bekräftigt. Die Ausübung praktischer medizinischer Tätigkeiten war auch ein wesentlicher Bereich der deutschen Kolonialexpansion in China. Währenddessen befassten sich manche Deutsche mit chinesischen medizinischhygienischen Gegebenheiten, woraus eine große Anzahl deutscher Publikationen zur chinesischen Medizin entstand. Zusammen mit der medizinischen Praxis bildeten diese Arbeiten das deutsche Medizinalwesen in China. Wie wurde die chinesische Medizin in diesen Arbeiten beschrieben und beurteilt? Was genau beeinflusste die Meinungsbildung der deutschen Beschreiber hinsichtlich der chinesischen Medizin? Auf diese Fragen ist bisher noch nicht systematisch eingegangen worden.

Obwohl die Tätigkeiten von Deutschen in China, vor allem deutscher Missionare und Kaufleute, vor den 1860er Jahren nicht durch staatliche Kräfte unterstützt wurden, erfolgten diese vor dem Hintergrund des europäischen Kolonialismus in China, der mit dem Opiumkrieg begann, und wiesen dieselbe Asymmetrie auf wie andere koloniale Beziehungen. Deshalb war ihr frühes Verhalten in China kolonialistisch gefärbt und sollte als Beginn der deutschen Kolonialexpansion in China angesehen werden. Nach dem Ende des Ersten Weltkriegs verlor Deutschland sämtliche koloniale Privilegien in China und ab Anfang der 1920er Jahren entwickelten sich vertraglich fixierte, gleichberech- 
tigte Beziehungen zwischen Deutschland und China, was einen neuen Beginn der deutsch-chinesischen Beziehungen symbolisierte. Auf Basis dieser Tatsachen kann der Zeitraum der deutschen Kolonialexpansion in China, nämlich der in der vorliegenden Studie als „Kolonialzeit“ bezeichnete, auf die Zeit zwischen dem Opiumkrieg und dem Ersten Weltkrieg begrenzt werden. In diesem Sinn werden Deutsche, die an der Gründung und Entwicklung des deutschen Kolonialunternehmens in China beteiligt waren, als „Kolonisatoren“ und Chinesen als „Kolonisierte“ betrachtet, aber nicht jeder „Kolonisator“, sondern nur ein Anhänger oder Vertreter des Kolonialismus wird als „Kolonialist“ bezeichnet.

Die vorliegende Studie ist eine überarbeitete Fassung der Arbeit, die im Wintersemester 2018/2019 vom Fachbereich Geschichts- und Kulturwissenschaften der Freien Universität Berlin als Dissertation angenommen und im Januar 2019 an die Hochschulschriftenstelle der FU abgegeben wurde. Wenn ich mich an das mehrjährige Studium an der Freien Universität Berlin erinnere, entsteht spontan ein Gefühl der Dankbarkeit.

Zunächst geht mein Dank an meinen Betreuer, Herrn Prof. Dr. Klaus Mühlhahn. Man kann sagen, dass ohne seine kontinuierlichen, geduldigen Hinweise diese Dissertation nicht hätte fertiggestellt werden können. Obwohl Prof. Mühlhahn sehr beschäftigt ist, fand er immer die Zeit, mit mir über meine Arbeit zu diskutieren. Von der Bereitstellung des Themas, der Recherche und Sammlung der Materialien, der Überarbeitung des Inhalts bis zur Festlegung der endgültigen Version begleitete er hilfreich das Entstehen der Dissertation. Hierfür bedanke ich mich bei Prof. Mühlhahn herzlich. Gleichzeitig bedanke ich mich auch bei Prof. Dr. Sun Lixin, dem Betreuer meiner Masterarbeit an der Pädagogischen Universität Beijing in China. Während ich an meiner Dissertation schrieb, gewährte er mir freundliche Unterstützung und große Hilfe. Er prüfte meine Arbeit und gab mir viele Hinweise und Überarbeitungsvorschläge. Daneben geht mein Dank auch an Prof. Dr. Mechthild Leutner, Prof. Dr. Christian Meyer und Prof. Dr. Paul Ulrich Unschuld, denen ich viele wertvolle Vorschläge zu verdanken habe.

Genauso großer Dank gebührt dem China Scholarship Council (CSC). Durch das Stipendienangebot des CSC wurde mir der mehrjährige Aufenthalt in Deutschland finanziell ermöglicht und die reibungslose Vollendung meines Studiums an der FU gewährleistet.

Bedanken möchte ich mich auch bei meinen Eltern und Freunden. Das Schreiben der Dissertation war ein langer Prozess und dauerte mehrere Semester. Während dieser Zeit sorgten sie sich immer um mich. Sie boten mir nicht nur materielle, sondern auch seelische Hilfen an, indem sie mich motivierten. Ihre Unterstützung war ein positiver Antrieb für das Vorankommen meiner Arbeit. 
Am Ende geht mein Dank an alle Leute, die mir im Laufe des Schreibprozesses in unterschiedlichen Graden mit Sorge, Hilfe und Unterstützung zur Seite standen.

Weiman Yuan

Tianjin, April 2020

\section{Titelsübersetzung chinesischer Literatur}

Für die Titel der chinesischen Literatur werden in dieser Arbeit deutsche Übersetzungen verwendet. Die englischen Übersetzungen der Titel stammen von den Literaturautoren selbst. 
